Ces deux dernières années, la Société suisse de médecine interne générale (SSMI) s'est - entre autre chose penchée sur la question de l'utilité de certains examens et traitements. Pour la première fois, elle publie une liste de tests et de traitements en médecine interne générale qui offrent peu de bénéfices pour les patients et auxquels il est généralement possible de renoncer. Ces évaluations de leurs propres pratiques par les médecins permettent des améliorations qui vont dans le sens du bien-être des patients. La FMH soutient cette initiative.

\title{
La SSMI lance sa campagne «Smarter Medicine» et la première liste «Top 5» de Suisse
}

Lors des Symposia Platin de la Société suisse de médecine interne générale (SSMI) à Thoune en 2012 et 2013, un panel d'orateurs, de représentants de médecins en pratique ambulatoire privée, des hôpitaux, des universités, de la FMH, de l'OFSP, de l'industrie pharmaceutique, des assurances maladies, du monde politique et des médias, ont débattu du thème «Can less be more?».

Ce thème a représenté l'enjeu de la «Choosing Wisely Initiative» [1], lancée aux USA en 2012, et de NICE, au RoyaumeUni, dès 1999 [2], bientôt reprises en Australie [3], en Italie et ailleurs. Dans ce cadre, un nombre important de disciplines médicales se sont lancées dans l'établissement d'une «Top-5 list», c'est-à-dire une liste de 5 investigations ou traitements à éviter, dont les évidences scientifiques montrent qu'ils n'offrent pas (ou peu) de bénéfices pour les patients, comportent des risques potentiels d'effets secondaires (notamment par les examens complémentaires ou les interventions qu'ils peuvent indûment induire) et sont pourtant fréquemment prescrits par les médecins des disciplines concernées.

Après avoir procédé à de riches échanges de vues sur le sujet à Thoune, la SSMI s'est sentie prête à relever ce défi et à se lancer, en pionnier, dans une initiative de type «Choosing Wisely» en Suisse. Cette démarche, intitulée «Smarter Medicine», s'inscrit dans la droite ligne de la prise de position de l'Académie Suisse des Sciences Médicales «pour un système de santé durable pour la Suisse» [4]. Elle rejoint également d'autres réflexions menées dans le monde sur la surutilisation en médecine, intitulées «too much medicine» [5].

\section{Les pratiques médicales suisses sont «smart»: peuvent-elles l'être encore plus?}

Un article de ce numéro présente la première liste Top 5 établie par la SSMI dans le domaine de la médecine interne générale ambulatoire. L'an prochain, une liste Top 5 sera publiée concernant des interventions pratiquées dans le cadre de la médecine interne générale hospitalière.
Comme vous le verrez dans ces textes, la SSMI se veut respectueuse du médecin et du malade. Le nom de sa campagne, «Smarter medecine», en atteste: nos pratiques médicales suisses sont «smart»; peuvent-elles l'être encore plus? Ainsi, la SSMI a émis des recommandations quant aux stratégies à éviter, à moins que les conditions cliniques ou le contexte individuel d'un patient, et de la relation qu'il entretient avec son médecin, ne dictent le contraire. A terme, ceci devrait contribuer à promouvoir le concept de décisions partagées entre médecins et patients.

\section{«To choose wisely» implique un dialogue entre médecin et malade.}

En effet, «to choose wisely» suppose un dialogue médecin-malade permettant cet échange. Ceci implique parfois, pour les médecins, un apprentissage de techniques d'entretien appropriées, et pour les patients, une responsabilisation vis-à-vis de leur maladie. Sur le plan des systèmes de santé, cela exige un large consensus et un effort de communication médiatique d'envergure. L'ambition de la SSMI est de contribuer positivement à ces efforts et ces débats.

Prof. Jean-Michel Gaspoz, président du directoire de la Société suisse de médecine interne générale

1 Choosing wisely: an initiative of the ABIM Foundation 2013. (www.choosingwisely.org).

2 Sarah Garner. Disinvestment form low value clinical interventions: NICEly done? BMJ. 2011;343:d4519 doi: 10.1136.

3 Elshaug AG, et al. Over 150 potentially low-value health care practices: an Australian study. MJA. 2012;197:556-60.

4 Un système de santé durable pour la Suisse. Feuille de route des Académies suisses des sciences. Académies suisses des sciences; 2012.

5 Glasziou P, et al. Too much medicine; too little care. Time to wind back the harms of overdiagnosis and overtreatment. BMJ. 2013;347:f4247. doi: 10.1136. 